International Journal of Applied Research 2018; 4(1): 47-50

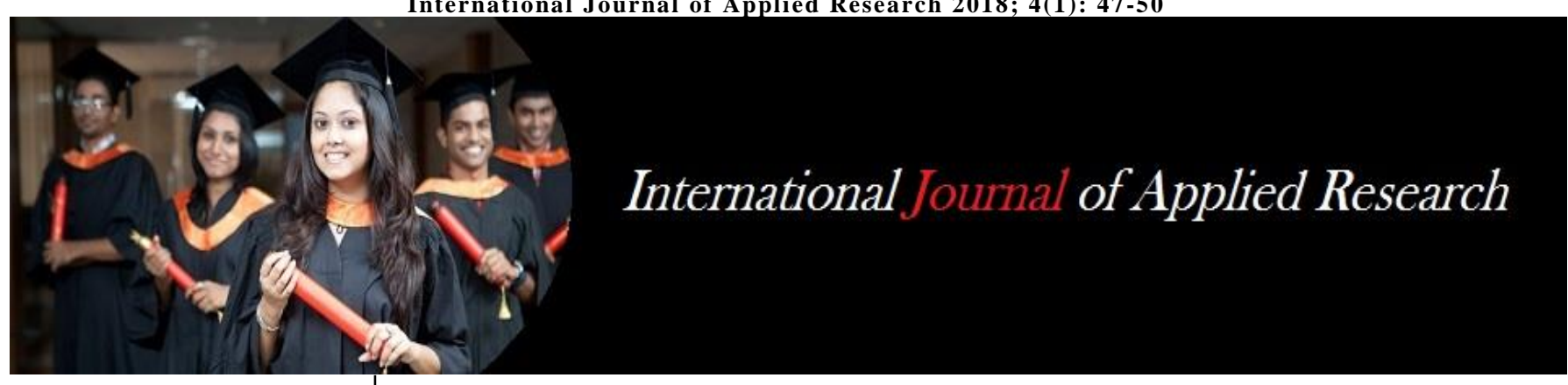

ISSN Print: $2394-7500$ ISSN Online: 2394-5869 Impact Factor: 8.4

IJAR 2018; 4(1): 47-50 www.allresearchjournal.com

Received: 24-11-2017 Accepted: 27-12-2017

रश्मि किरण

शोधार्थी, इतिहास विभाग, ल.ना.मिथिला विश्वविद्यालय, दरभंगा, बिहार, भारत।
Corresponding Author: रश्मि किरण शोधार्थी, इतिहास विभाग, ल.ना.मिथिला विश्वविद्यालय, दरभंगा, बिहार, भारत।

\section{महिला सशक्तिकरण हेतु बालिका शिक्षा की आवश्यकताः एक ऐतिहासिक अध्ययन}

\section{रश्मि किरण}

DOI: https://doi.org/10.22271/allresearch.2018.v4.i1a.7476

\section{सारांश}

किसी भी समस्या के निराकरण हेतु हमें उसकी जड़ को पहचानना पड़ता है। आज यदि नवजात बच्ची से लेकर प्रोढ़ महिला तक को दोयम दर्जे का शिकार होना पड़ रहा है तो उसका कारण एक तरफ लोगों के संकीर्ण मानसिकता है तो वहीं दूसरी तरफ उनकी दयनीय आर्थिक स्थिति भी इसकी बराबर की जिम्मेदार है। अतः हमारे प्रयास दोनों ही दिशाओं में एक साथ होने चाहिए। बालिकाओं के विकास व शिक्षा में आने वाली आर्थिक बाधा को दूर करने वाली वैकल्पिक योजनाओं के साथ-साथ जनमानस के अंतर्मन से बालिकाओं व महिलाओं के लिए बनाई गई संकीर्ण अवधारणाओं की समाप्ति भी आवश्यक हैं इसलिए हमें 'बेटी पढाओं एवं 'सुकन्या समृद्धि योजना' के साथ-साथ पूर्व की 'सशर्त नकद हसतांतरण' योजनाओं का सरलीकरण कर, इनमें व्याप्त विसंगतियों को दूर कर कार्यान्वित करना होगा। सभी सतरों पर बेहतर तालमेल व समग्र प्रयास के द्वारा ही देश में बेटियों की दशा सुधर पाएगी।

\section{प्रस्तावनाः}

भरतीय संसकृति एवं परंपरा में "नारी नारायणी", एवं "यत्र नार्यस्तु पूज्यनते रमन्ते तत्र देवता:" कहकर नारी को विशेष सम्मान दिया गया है और उसके महत्त्व को रेखांकित किया गया है। महिलाओं को दिए जाने वाले सम्मान व स्त्रियों के प्रति हमारी संस्कृति की आस्था व समर्पण भाव से हम सभी अवगत है। लेकिन इन आदर्शात्मक बातों से दूर धरातल की सच्चाई कुछ और ही स्तिथि को बयां करती है। जन्म लेने के पूर्व से लेकर जन्म लेने, किशोरावस्था, व प्रोढ़वस्था तक हर कदम पर भिन्न प्रकार की चुनौतियों से दो-चार होती इस देश की महिला आबादी अपने सह-असिततव को लेकर संघर्षरत है। अतः हमें भी चाहिए कि कर्णप्रिय आदर्शात्मक पंक्तियों की दुनिया से बाहर निकल वास्तव में समाज में जो बालिकाओं व महिलाओं की दयनीय स्थिति है, उसे स्वीकार किया जाए तथा इससे मुक्ति पाने के रास्ते तलाशे जाएँ।

'नारी तू नारयणी की बात करने वाले इसी भारत देश में 1961 से लेकर अब तक बाल लिंग अनुपात जो 0 से 6 वर्ष की उम्र तक के प्रति एक हजार बालकों की तुलना में बालिकाओं की संख्या दर्शाता है, घटता जा रहा हैं और 2011 की जनगणना के अनुसार यह आँकड़ा 919 पर आ गिरा है। इस प्रकार बालिकाओं के घटते अनुपात दीर्घकाल में हमारे जनसांख्यकीय संरचना के लिए एक खतरनाक स्थिति का निर्माण कर सकते है। हमारे यहाँ पुरूष साक्षरता की तुलना में महिला साक्षरता लगभग 16 प्रतिशत कम है (पुरूष साक्षरता-82.14: महिला साक्षरता 65.46:) सांख्यिकी एवं कार्यक्रम कार्यान्वयन मंत्रालय (MOSPI) की रिपोर्ट के अनुसार 63.5: लड़कियाँ बीच में ही स्कूली शिक्षा छोड़ देती हैं।

राष्ट्रीय पारिवारिक स्वास्थ्य सर्वेक्षण (NFHS) की तीसरी रिपोर्ट के अनुसार 47: किशोरावस्था (15-19 वर्ष की आयु वर्ग) बालिकाएँ कम वजन की समस्या अर्थातृ जिनका बॉडी मास इंडेक्स 18.5 किग्रा से कम हे, से ग्रसित हैं। और 56: किशोर बालिकाएँ एनीमिया की समसे ग्रसित हैं। भारत में लिंग भेद की भीषण स्थिति को इसी से समझा जा सकता है कि यू.एन.डी.पी. द्वारा जारी किये जाने वाले मानव विकास सूचकांक 2014 में भारत 188 देशों में 130 वें स्थान पर था। रिपोर्ट के अनुसार यदि इसमें से पुरूषों को बाहर कर सिर्फ महिलाओं को रखा जाए तो भारत 151 वें स्थान पर होता। उपरोक्त चंद आंकड़े यह दर्शाने के लिए काफी हैं कि जो देश तीव्र विकास करने व दहाई अंक की विकास दर को प्राप्त करने की नीतियाँ बनाने में व्यस्त हैं वहाँ की आधी आबादी किस प्रकार विकास से महरूम है। 
कहते हैं कि किसी भी समस्या के निराकरण हेतु हमें उसकी जड़ को पहचानना पड़ता है। आज यदि नवजात बच्ची से लेकर प्रौढ़ महिला तक को दोयम दर्जे का शिकार होना पड़ रहा है तो उसका कारण एक तरफ लोगों की संकीर्ण मानसिकता है तो वहीं दूसरी तरफ उनकी दयनीय आर्थिक स्थिति भी इसकी बराबर की जिम्मेदार है। आज यदि किसी गरीब या फिर निम्न मध्यमवर्गीय परिवार की बात करें तो वह सदैव अपने सीमित आर्थिक संसाधनों के प्रयोग के लिए वरीयता अपने पुत्र को देगा, चाहे प्रश्न उसकी शिक्षा को हो या अन्य आवश्यकताओं का। बेटी को बोझ व पराया धन जैसे शब्दों से आज भी नवाजा जाता है, और जन्म के साथ ही परिवार खुद को बोझिल महसूस करने लगता है। यही कारण है कि समाज का एक बड़ा धड़ा तो तकनीकी का दुरूपयोग कर लिंग जानने और फिर कन्या भूण हत्या जैसे जघन्य कुकृत्य को अंजाम देने में भी नही हिचकता। अतः हमारे प्रयास दो दिशाओं में एक साथ होने चाहिए-

- बलिकाओं के विकास व शिक्षा में आने वाली आर्थिक बाधा को दूर करने वाली वैकल्पिक योजनाएँ

- लोगों के अंतर्मन से बालिकाओं व महिलाओं के लिए बनाई गई संकीर्ण अवधारणा की समाप्ति

हालांकि हमारी सरकारों द्वारा उपरोक्त दोनों ही दिशाओं मे कई प्रकार की योजनायें चलाई जाती रही हैं, केन्द्र व राज्य सरकारें की योजनाओं को मिलाकर देखा जाए तो अच्छी-खासी संख्या बन जाती है। परंतु ये सभी वांछित परिणाम क्यों नहीं दे पए, इसका विश्लेषण भी हम आगे करने का प्रयास करेंगे। अब यदि वर्त्तमान केन्द्र सरकार की बात की जाए तो प्रधानमंत्री श्री नरेन्द्र मोदी की नेतृत्व वाली इस सरकार ने भी समाज में बालिकाओं की स्थिति सुधारने को लेकर अपनी प्रतिबद्धता जाहिर करते हुए एक नई योजना "बेटी बचाओं, बेटी पढ़ाओ" को प्रारंभ किया है। बालिकाओं के अस्तित्व एवं सुरक्षा को सुनिश्चित करना इस योजना के मुख्य उद्देश्य हैं। यह योजना महिला एवं बाल विकास मंत्रालय सहित परिवार कल्याण मंत्रालय एवं मानव संसाधन विकास मंत्रालय की संयुक्त पहल है। प्रथम चरण में इस योजना को निम्न बाल लिंग अनुपात वाले 100 जिलों में प्रारभ किया गया है तथा इनके चयन के तीन मानदंड है-

- राष्ट्रीय ओसत से कम बाल लिंग अनुपात वाले ऐसे 87 जिलों का चयन जो कि 23 अलग-अलग राज्यों से होंगे।

- आठ राज्यों से आठ जिलो का चयन किया गया है जहाँ अनुपात तो राष्ट्रीय औसत के समान है परंतु बराबर गिरावट का रूख है।

- पाँच राज्यों के पाँच जिलों का चयन जहाँ बाल लिंग अनुपात राष्ट्रीय औसत से अधिक भी हो और जिन्होंने या तो अपने लिंगानुपात के स्तर को बनाए रखा अथवा उसमें बढ़ोत्तरी दर्ज की है। इन जिलों के चयन का कारण यह है कि इनसे सीख लेकर बाकी जगहों पर इसे दोहराया जा सके।

नि:संदेह इस प्रकार के चयन की प्रक्रिया तार्किक व वैज्ञानिक लगती है। वहीं दूसरी तरफ यह योजना 'नकद हस्तांतरण' योजनाओं से अलग जन-जागरूकता अभियान के द्वारा बेटियों के संरक्षण, स्वास्थ्य एवं शिक्षा को सामाजिक आंदोलन का रूप देने की दिशा में प्रयासरत दिखती है। हालाकि इस योजना की क्या सीमाएँ व चुनौतियाँ होंगी, इस पर भी हम आगे बात करेंगे।

\section{वित्तिय संसाधन:-}

'बेटी बचाओ बेटी पढ़ाओं' योजना के लिए वर्त्तमान वर्ष में 100 करोड़ रूपये का बजट आवंटन किया गया है। योजना के बृहद सतर और प्रारूप को देखते हुए यह यक्ष प्रश्न है कि क्या यह राशि पर्याप्त होगी इसकी आवश्यकताओं को पूर्ण करने हेतु। हमने पूर्व में भी देखा है अच्छी योजनाएँ भी वित्तिय अभाव में बंद करनी पड़ती हैं। इसका एक अच्छा उदाहरण राजस्थान सरकार
की 'राजलक्ष्मी' योजना है। अनुमानतः राजस्थान बालिकाओं की स्थिति सुधारने के लिए 'सशर्त नकद हस्तांतरण योजना' लाने वाला पहला राज्य था जिसने 1992 में 'राजलक्ष्मी' योजना प्रारंभ की भी जिसे 1996 में पुनः कुछ नियमों को उदार कर प्रस्तुत किया गया था। यह कार्यक्रम राजस्थान सरकार एवं यूनिट ट्रस्ट ऑफ इंडिया (UTI) के तालमेल से तैयार किया गया था जिसमें पाँच वर्ष से कम आयु की बच्ची के नाम राजस्थान सरकार द्वारा 1500 रूपये जमा किये जाते थे जो 21 वर्ष की लॉक-इन-अवधि के पश्चात 2100 रूपये के रूप में मिलते थे। यह योजना अच्छा कर रही थी और हरियाणा, कर्नाटक जैसे राज्यों ने इसी योजना का अनुसरण कर अपने यहाँ भी इस प्राकर की योजनाएँ बनाई। "राजलक्ष्मी योजना" में लाभार्थियों की संख्या वर्ष 1992-93 में 4917 से बढ़कर वर्ष 1997-98 में 11664 तक पहुँची थी, जो इसको शनै: सफल भी होने का संकेत देती है, परंतु बीच में ही वर्ष 2000 में वित्तिय संसाधन के अभाव में सरकार को इस योजना को बंद करना पड़ा था।

अतः इससे सीख लेते हुए हमारी सरकारों को किसी भी कल्याणकारी योजना को प्रारंभ करने से पहले आवश्यक वित्तीय संसाधनों की उपलबधता अवश्य सुनिश्चित करनी चाहिए।

इस बार के बजट को देखा जाए तो सरकार की कथनी एवं करनी में विरोधाभास दिखता है। वित्तीय वर्ष 2015-16 के लिए महिला एवं बाल विकास मंत्रालय को आवंटित राशि में पिछले वर्ष की तुलना में लगभग 50 प्रतिशत की कटौती दिखती है। जहाँ वर्ष 2014-15 के बजट में नियोजित परिव्यय हेतु प्रस्तावित राशि 21100 करोड़ थी वहीं वर्ष 2015-16 के बजट में नियोजित परिव्यय की राशि घटाकर 10286.73 करोड़ कर दी गई है। वर्त्तमान वित्तीय वर्ष के लिए आवंटन का सबसे बड़ा हिस्सा "समेकित बाल विकास सेवा ;प्ब्रेद्ध पर गया है यह राशि 8477.77 करोड़ रूपये है। यह योजना एक अत्यंत ही बृहद योजना है जिसके अन्तर्गत लगभग 7076 परियोजनाएँ कार्यान्वित हो रही है। लगभग 17 लाख आंगनबाड़ी केन्द्रों के माध्यम से 0-6 वर्ष तक के बच्चों एवं गर्भवती महिलाओं को पूरक पोषक तत्त्व, बीमारियों से प्रतिरक्षा हेतु टीका इत्यादी के साथ-साथ नियमित स्वास्थ्य जाँच की व्यवस्था होती है। इसके अतिरिक्त 0-6 वर्ष तक के बच्चों एवं बच्चियों को स्कूल-पूर्व की शिक्षा भी यहाँ से उपलब्ध कराई जाती है। इतने वृहद स्तर की परियोजना की राशि भी पिछले वित्तीय वर्ष की तुलना में आधी कर दी गई है, वहीं किशोरी बालिकाओं से संबंधित योजना जिसे 'सबला" के नाम से भी जाना जाता है, के लिए महज 75.50 करोड़ रूपये आवंटित किये गए हैं। 11-18 वर्ष की किशोरावस्था बालिकाओं के विकास से संबंधित इस योजना को वर्ष 2010 में प्रारंभिक किया गया था। यह वर्त्तमान में 205 जिलों में कार्यान्वित हो रही है। इस योजना के दो घटक हैं-पहला-पोषक तत्त्व व भोजन की उपलब्धता तथा दूसरा-स्वस्थय जाँच, स्वास्थ्य संबंधी शिक्षा, परिवार कल्याण, सैक्युअल हेल्थ संबंधी विषयों पर मार्गदर्शन एवं परामर्श सहित व्यावसासिक शिक्षा आदि का प्रबंध। अगर उपरोक्त बिन्दुओं को देखा जाए तो 'सबला' एक अत्यंत महत्वपूर्ण योजना मालूम पड़ती है परंतु इसमे भी वित्तीय कटौती किया जाना यह सोचने पर विवश करता है कि कहीं यह कदम इस योजना के लक्ष्य को साधनों में बाधा न बन जाए। इन केन्द्र प्रायोजित योजनाओं में हो रही वित्तीय कटौती के कारण केन्द्र सरकार द्वारा 14वें वित्त आयोग की अनुशंसा को मानते हुए केनद्रीय करों में राज्यों की हिस्सेदारी को बढ़ाकर 42: कर देना हो सकता है, जिसके बाद केन्द्र सरकार केन्द्र प्रायोजित योजनाओं को लेकर राज्य सरकारों को मुख्य भूमिका में आने की अपेक्षा कर रह है। खैर, यह कदम व सोच किस हद तक सफल होंगे यह तो भविष्य के गर्भ में है।

\section{सशर्त नकद हस्तांतरण योजनाः}


हमने प्रारंभ में भी जिक्र किया था कि विभिन्न सरकारों द्वारा बालिकाओं के संरक्षण व विकास को सुनिश्चित करने के उद्देश्य से नकद हस्तांतरण योजनाएँ भी चलाई जाती रही हैं। वर्ष 2008 में भारत सरकार के महिला एवं बाल विकास मंत्रालय द्वारा "धनलक्ष्मी" योजना को सात राज्यों के ग्यारह प्रखंडों में प्रारंभ किया गया। इस योजना के अन्तर्गत 19 नवम्बर , 2008 के बाद जनम लेने वाली बालिका के लिए बीमा योजना की व्यवस्था थी तथा 18 वर्ष तक युवती के अविवाहित रहने पर, बीमा अवधि की समाप्ति के पश्चात् एक लाख रूपये मिलते। इसके साथ ही बालिका के परिवार को और कई बिन्दुओं की पूर्ति पर भी नकद हस्तांतरण का प्रावधान था। जैसे जन्म के समय पंजीकरण के पश्चात् 5000 रूपये, फिर टीकाकरण के समय, प्राथमिक विद्यालय में दाखिले के समय, आठवीं तक स्कूली शिक्षा पूरी करने तक इत्यादि। हालांकि लक्ष्य को प्राप्त करने एवं आवंटित किये गए फंड के प्रयोग के आधार पर मूल्यांकन किया जाए तो यह योजना सफल साबित नही हुई। वर्ष 2008-09 के लिए लाभान्वित होने वाली बालिकाओं का लक्ष्य एक लाख था जवकि महज 79555 बालिकाएँ ही पंजीकृत हुईं एवं आवंटित 10 करोड़ में से 5.95 करोड़ खर्च हो पाए। दूसरे वर्ष के लिए भी स्थिति असंतोशजनक ही रही।

इसी से मिलती-जुलती मध्यप्रदेश सरकार की योजना है 'लाडली लक्ष्मी योजना' जिसे मध्य प्रदेश सरकार द्वारा 2007 में प्रारंभ किया गया था जिसके अंतर्गत राज्य सरकार एक बच्ची के जन्म पर 6000 रूपये के राष्ट्रीय बचत पत्र में निवेश करती है। इससे मिलने वाली राशि बालिक के कक्षा छठी, नौवीं व ग्यारहवीं के शुल्क हेतु प्रयोग में आती है, तथा बची हुई राशि 21 वर्ष की आयु पर बालिका को मिल जाती है। इस प्रकार इससे शिक्षा व वित्तीय सुरक्षा दोनों लक्ष्य पूर्ण होते है यह योजना काफी जनप्रिय हुई तथा अन्य कई राज्यो ने इसका अनुसरण भी किया गया है, कुछेक भ्रष्टाचार की समस्या तथा प्रक्रियात्मक जटिलता की शिकायतें जरूर आई इसके संदर्भ में।

उपरोक्त दोनों उदाहरणों से स्पष्ट है कि इन नकद हस्तांतरण कार्यक्रमों से वांछित लाभ लेने के लिए हमें इनकी विसंगतियों को दुरूस्त करना होगा।

\section{चुनौतियाँ :}

योजना आयेग के लिए तैयार की गई टी.वी. शेखर की समीक्षात्मक रिपोर्ट में देशभर में बालिकाओं के लिए चलाए जा रहे विशेष वित्तीय सहयोग संबंधी 15 योजनाओं का विस्तार से अध्ययन कर उनमें आने वाली चुनौतियाँ तथा विसंगतियाँ जिनकी वजह से हम इन योजनाओं से आशा अनुरूप परिणाम नही प्राप्त कर पा रहे, तो चिन्हित किया गया है जिनमें कुछ निम्नलिखित है

\section{योजना की शर्तेः}

योजना की अनेक प्राकर की शर्तें तथा जटिल प्रक्रिया जैसे जन्म के साथ ही पंजीकरण, इसके अलावा ज्यादातर योजनाओं के लिए एक या दो बालिका संताना का ही होना जनता में ऐसे कार्यक्रमों की जटिलता बढ़ाता हैं इन योजनाओं का एक बड़ा नकारात्मक पक्ष यह रहा कि इन्हें परिवार नियोजन से जोड़ने का प्रयास किया गया है। कई योजनाओं में दंपत्ति के द्वारा नसबंदी या बंध्याकरण की शर्तने भी योजना में नकारात्कय योगदान किया। योजनाओं में केवल बाकि संतान की आवश्यकता ने भी योजना की स्वीकार्यता को घटाया। अतः बालिका उत्थान से संबंधित योजना को परिवार नियोजन से जोड़ना गलत प्रयास था।

- ज्यादातर योजनाएँ गरीबी रेखा से नीचे के लोगों तक ही सीमित रहीं। योजनाओं में बीपीएल परिवारों को ही भागीदारी देना भी सही कदम नहीं रहा क्योंकि यह समस्या केवल गरीब या गाँव तक की सीमित नहीं हैं।
- तलमेल का आभावः योजना के कार्यान्वयन में विभिन्न विभागों के बीच समन्वय व तालमेल का अभाव भी एक बड़ी चुनौती है। इन योजनाओं का कार्यान्वयन बृहद् तंत्र के द्वारा होता है जिसमें लाखों आंगनबाड़ी शामिल होते हैं। केन्द्र व राज्य सरकारों के बीच तालमेल, विभिन्न मंत्रालयों जैसे, शिक्षा, स्वास्थ्य, महिला एवं बाल विकास इन सभी के बीच भी समन्वय की आवश्यकता होती हैं इसके अतिरिक्त वित्तीय संस्थानों जैसे एल.आई.सी., बैंक इत्यादि से भी तालमेल की आवश्यकता होती हैं इन सबके बिना योजनाओं का सफल संचालन नहीं हो सकता। वर्त्तमान सरकार द्वारा प्रारंभ की गई 'बेटी बचाओं बेटी पढ़ाओं' योजना में भी सबसे बड़ी चुनौती तालमेल को लेकर ही आ सकती है।

- अवसंरचना का अभाव : टी.वी. शेखर की रिपोर्ट यह भी मानती है कि योजनाओं के कार्यान्वयन हेतु आवश्यक अवसंरचना के निर्माण के लिए अत्यंत कम राशि खर्च की जाती है तथा ज्यादातर योजनाएँ आईसीडीएस के तंत्र का प्रयोग करती हैं जिससे इस पर दबाव बढ़ता है तथा ज्यादातर संदर्भों में पंचायतों, क्षेत्रीय एन.जी.ओ. एवं महिलाओं के स्वयं सहायता समूहों की अत्यंत सीमित भूमिका होती है जबकि इन सबकी विसतृत व प्रबल भूमिका होनी चाहिए।

अतः अब यह स्पष्ट है कि 'नकद हस्तांतरण' से संबंधित ये योजनाएँ अधिक प्रभावशली हो सकती हैं बशर्त इनका सरलीकरण कर, इनमें व्याप्त विसंगतियों को दूर कर इनको कार्यान्वित किया जाए।

इन योजनाओं का हमारी अर्थव्यवस्था पर एक अन्य सकारात्मक प्रभाव भी पड़ सकता है। सरकार द्वारा इन योजनाओं के लिए भिन्न प्रकार की बीमा पॉलिसी, राष्ट्रीय बचत-पत्रों, या बॉण्ड इत्यादि में निवेश करने से वित्तीय बाजार में पूंजी का अंतर प्रवाह होता है, जिससे वित्तीय संस्थानों की ऋण देने की क्षमता में वित्तीय बाजार में वृद्धि हो सकती है और इसके गुणक प्रभाव स्वरूप अर्थव्यवस्था में निवेश बढ़ जाएगा जिसका आर्थिक विकास की प्रक्रिया पर भी एक सकारात्मक प्रभाव पड़ेगा।

\section{महिला सशक्तीकरण के कानून और कार्यक्रम :}

महिला सशक्तीकरण हेतु संसद द्वारा महिलाओं के अधिकारों के संरक्षण के लिए समय-समय पर कानूनों का प्रावधान किया गया है जिसमें समान पारिश्रमिक अधिनियम 1976, प्रसूति प्रसुविधा अधिनियम 1961, बाल विवाह निषेध अधिनियम 1976, वेश्यावृत्ति निवारण अधिनियम 1986, सती प्रथा निरोधक अधिनियम 1987, प्रसव-पूर्व निदान तकनीकी अधिनियम 1994, गर्भ का चिकित्सकीय समापन अधिनियम 1971, अनैतिक व्यापार निरोधक 1959 (1986 में संशोधन), घरेलू हिंसा रोकथाम अधिनियम 2005, हिन्दू उत्तराधिकार संशोधन अधिनियम 2005 आदि महिलाओं के अधिकारों की रक्षा के लिए बनाए गए।

केन्द्र सरकार द्वारा महिला सशक्तीकरण की दिशा में कई कार्यक्रम एवं योजनाओं को भी चलाया गया है, जिसमें समेकित बाल विकास योजना 1975, स्वावलम्बन योजना 1982, महिला समाख्यान योजना 1989, पुर्नउत्पादित एवं बाल स्वास्थ्य योजना 1997, बालिका समृद्धि योजना 1997, महिलाओं एवं बालिकाओं के लिए विश्रामगृह योजना 1999, किशोरी शक्ति योजना 2000, श्री शक्ति पुरस्कार योजना 2000, स्वधार योजना 2001, सर्व शिक्षा अभियान योजना 2001, जीवन भारती महिला सुरक्षा योजना 2003, कस्तूरबा गाँधी विशेष बालिका विद्यालय योजना 2004, उज्ज्वल योजना 2005, राष्ट्रीय ग्रामीण स्वास्थ्य मिशन योजना 2005, बालिका प्रोत्साहन योजना 2006, जन्नी सुरक्षा योजना 2006, इंदिरा गांधी इकलौती कन्या छात्रवृति योजना 2006, इंदिरा गांधी राष्ट्रीय निशक्तता प्रेंशन योजना 2007, प्रियदर्शनी परियोजना 2008, राष्ट्रीय स्वास्थ्य बीमा योजना 2008, समेकित 
बाल संरक्षण योजना 2009, इदिंरा गांधी मातृत्व सहयोग योजना 2010, सबला योजना 2012, कार्यरत महिला हॉस्टल योजना 2013, स्वयंसिद्धा योजना 2013 सम्मिलित हैं।

\section{समाज के हर तबके को जोड़ने की आवश्यकता}

पुनः यदि मूल मुद्दे पर आएँ तो हम पाएँगे कि ऐसा नहीं है कि बेटियों को लेकर उदासीनता महज आर्थिक रूप से विभिन्न परिवारों में ही सीमित है, बल्कि निम्न अध्यमवर्गीय या मयमवर्गाय परिवार, जो भले ही आर्थिक रूप से ठीक हों, पर मानसिक अवधारणा यहाँ भी बेटियों को बोझ मानने की ही है।

अतः समाज के हर तबके को समान रूप से प्रोत्साहित करने की आवश्यकता है। और ऐसे वर्ग के लिए सरकार द्वारा लाई गई 'सुकन्या समृद्धि योजना' अत्यंत कारगर हो सकती है। इस योजना के तहत वर्त्तमान के सर्वाधिक 9.2: ब्याज दर का प्रावधान है, तथा इनमें निवेश का लाभ आयकर में धारा 80 सी के तहत छूट के रूप में भी मिलेगा। इस योजना के तहत 10 वर्ष की उम्र तक की बच्ची का खाता खोला जा सकता है। यह खाता मात्र 1000 रूपये से खोला जा सकता है, तथा प्रति वर्ष न्यूनतम 1000 रूपये व अधिकतम $1,500,000$ रूपये तक इस खाते में जमा किए जा सकते हैं। इसमें खाता खोलने से अगले 14 वर्ष तक प्रतिवर्ष राशि जमा करनी है। तथा खाता खोलने की तिथि से 21 वर्ष बाद यह परिपक्व होगा। हालांकि इससे पूर्व भी बालिका के 18 वर्ष पूरे हो जाने की स्थिति में उसकी शिक्षा या विवाह हेतु 50 प्रतिशत तक राशि निकाली जा सकती है। तथा विवाह हो जाने की स्थिति में खाते को बंद करना होगा।

इस योजना के दो महत्वपूर्ण आकर्षक पहलू हैं-पहला, इस पर वर्त्तमान में मिलने वाली सर्वाधिक ब्याज दर तथा दूसरा इसमें निवेश से मिलने वाली छूट। इस योजना के अंतर्गत जमा की गई राशि 80 सी के तहत टैक्सेबल इनकम से डिडक्अ होगी (अधिकतम सीमा $1,50,000$ तक) तथा इसमें मिलने वाले ब्याज व योजना के परिपक्व होने पर मिलने वाली राशि पर भी आयकर से छूट प्राप्त होगी। उपरोक्त दोनों ही बिन्दु सामान्य परिवारों को इस योजना में अपनी छोटी-मोओ बचत का निवेश करने को उत्प्ररित करेंगी। तथा इससे उनके मन में बेटियों को लेकर जो तनाव व असुरक्षा का भाव रहता है, वा भी दूर होगा। हालांकि इस योजना के कुछ और बिन्दुओं पर भी थोड़ा ध्यान दिया जाए तो यह और आकर्षक हो सकती है। जैसे इसकी परिपक्वता अवधि (खाता खुलने से 21 वर्ष तक) काफी अधिक है, अन्ययोजनाओं की तरह यह भी दो पुत्रियों तक ही सीमित है (तीन, यदि दूसरी बेटी के जन्म के समय जुड़वां बच्चियाँ हुईं), अभी तो इसका ब्याज दर बाजार में अधिकतम है, परंतु भविष्य में इसको लेकर निश्चिता नहीं है। बेहतर होता यदि कोई न कोई न्यूनतम ब्याज दर भी तय कर दी जाती तो लोगों के मन में निश्चिता का भाव रहता। खैर, ये सुधार आगे भी हो सकते हैं परंतु कुल मिलाकर यह एक अभिनव प्रयास है जिससे सकारात्मक प्रभाव की पूरी अपेक्षा है।

निष्कर्षतः हम यह कह सकते हैं कि सशक्तीकरण की दिशा में पहल के साथ ही जन-जागरण भी किया जाए तो निश्चित ही इस राष्ट्र में बेटियों की दशा सुधरेगी।

\section{संदर्भ}

1. सुनीता सिंह (2003), वोमेन लीडारशिप इन ऑर्गानाईजेशन सोसियो कल्वरल डीटरमीनेन्ट, ज्ञान पब्लिशिंग हाउस, पृ० 254

2. बी० एम0 मिश्रा (2004), वोमेन एण्ड एडुकेशन, कॉमनवेल्थ पब्लिकेशन, नई दिल्ली, पृ० 378
3. लीना डोमीनेल (2007), वोमेन एण्ड कम्युनिटी एक्शन, रावत पब्लिकेशन, नई दिल्ली, पृ० 104

4. सुमित्रा कुमारी (2006), डायनामिक्स ऑफ वोमेन इम्पावरमेनट, अल्फा पब्लिकेशन, नई दिल्ली, पृ० 340

5. कांति शर्मा (2014), वोमेन पावर इन दी ट्वेनटी फस्ट सुन्चुरी, नई दिल्ली, अनमोल प्रकाशन, पृ० 426

6. रमन बिहारी लाल-शिक्षा के दार्शनिक एवं समाज शासत्रीय आधार 2010, रस्तोगी पब्लिकेशन मेरठ

7. कुरूक्षेत्र सितम्बर 2010 , प्रकाशन विभागे, नई दिल्ली कुरूक्षेत्र (अंग्रेजी संस्करण) सितम्बर 2012 प्रकाशन विभाग, नई दिल्ली

8. कुरूक्षेत्र सितम्बर, 2011, अंग्रेजी संस्करण, प्रकाशन विभाग, नई दिल्ली

9. कुरूक्षेत्र जनबरी, 2016

10. योजना, नवम्बर, 2019 